\title{
Early treatment failure in concurrent dengue and mixed malaria species infection with suspected resistance to artemisinin combination therapy from a tertiary care center in Delhi: a case report
}

\author{
This article was published in the following Dove Press journal: \\ International Medical Case Reports Journal \\ 16 August 2017 \\ Number of times this article has been viewed
}

\section{Rushika Saksena' \\ Monika Matlani' \\ Vineeta Singh ${ }^{2}$ \\ Amit Kumar ${ }^{2}$ \\ Anupam Anveshi' \\ Dilip Kumar ${ }^{3}$ \\ Rajni Gaind'}

'Department of Microbiology, Vardhman Mahavir Medical College and Safdarjung Hospital, Delhi, India; ${ }^{2}$ Cell Biology Laboratory and Malaria Parasite Bank, National Institute of Malaria Research, Delhi, India; ${ }^{3}$ Department of Internal Medicine, Vardhman Mahavir Medical College and Safdarjung Hospital, Delhi, India

Correspondence: Monika Matlani Department of Microbiology, Vardhman Mahavir Medical College and Safdarjung Hospital, 205 A MIG Flats Rajouri Garden, Delhi II 0029 , India

Tel +9| 9582691920

Email monikamatlani@yahoo.com

Vineeta Singh

Cell Biology Laboratory and Malaria Parasite Bank, National Institute of Malaria Research, Sector 8, Dwarka, Delhi I 10077, India

Email vineetas_2000@yahoo.com
Background: Concurrent dengue and mixed malaria infections in a single patient present with overlapping clinical manifestations which pose a diagnostic challenge and management dilemma in areas of common endemicities.

Methods: We report a case of a young male who tested positive for both Plasmodium vivax and Plasmodium falciparum along with dengue infection. He showed signs of early treatment failure to artemisinin combination therapy (artesunate with sulfadoxine+pyrimethamine). Molecular analysis for the drug resistance genes viz: chloroquine resistance $(p f c r t)$, multidrug resistance ( $p f m d r-1)$, sulfadoxine ( $p f d h p s)$, pyrimethamine (pfdhfr), and artemisinin resistance (keltch 13) was performed.

Results: A rise in parasitemia from $<2 \%$ to $5 \%$ was observed after 3 days of treatment. Mutations in $p f c r t, p f m d r-1, p f d h f r$, and $p f d h p s$ genes were detected as a possible cause of treatment failure.

Conclusion: Increased severity, overlapping symptoms, and suspected resistance to treatment warrants a multidimensional diagnostic approach and diligent therapeutic monitoring.

Keywords: dengue, drug resistance genes, mixed malaria infections, treatment failure

\section{Introduction}

Dengue and malaria both are major public health problems in India, and they share similar epidemiology. ${ }^{1,2}$ Delhi is hyperendemic for dengue, while for malaria, it is a low-transmission region. Though the percentage of malaria cases attributed to P. falciparum has increased from 39\% in 1995 to $65 \%$ in 2014 in India, the incidence of mixed Plasmodium species infection remains undetermined, especially in regions of low endemicity and transmission. ${ }^{1}$ It has been well established that concurrent infections of dengue and malaria present with severe manifestations; however, the effect of coinfections on drug resistance still needs to be investigated. We report here a patient who presented with concurrent infections of dengue, $P$. vivax, and $P$. falciparum with suspected resistance to artemisinin combination therapy (ACT), highlighting the importance of multidimensional diagnostic approach and therapeutic monitoring even in low-transmission regions. ${ }^{1}$ The Institutional Ethical Committee of Vardhman Mahavir Medical College and Safdarjung Hospital deemed the study exempt from ethical approval as the case was part of routine diagnostic work and no additional intervention was performed. 


\section{Case}

A 17-year-old male presented to the hospital emergency with fever associated with chills for the past 5-6 days. He also had complaints of abdominal pain and vomiting. The patients did not have any complaints of rash, joint pains, cough, difficulty in breathing, and any feature suggestive of urinary tract infection. The history of seizures and bleeding from any site was ruled out. There was no past history of diabetes mellitus, hypertension, tuberculosis, smoking, or alcohol intake. The patient did not give a history of previous treatment or self-medication. The patient resided in a slum area in east Delhi with a drain near his house.

On examination, the patient was sick and his general condition was poor. He was conscious and oriented and had severe pallor. His heart rate was 110 beats/min, respiratory rate was 16 breaths/min, and blood pressure was $110 / 70$ mmHg. On per abdominal examination, abdomen was soft and nontender. Hepatomegaly and massive splenomegaly was detected. On central nervous system examination, patient was lethargic, but no other abnormality was present. On chest and cardiovascular examination, no significant abnormality was detected. On the basis of these findings, a provisional diagnosis of acute undifferentiated fever was made with a differential diagnosis of malaria, dengue fever, and enteric fever. The patient was admitted in the department of internal medicine, and the case was further investigated.

The hemoglobin of the patient was $5 \mathrm{~g} / \mathrm{dL}$, total leukocyte count was $3,900 / \mathrm{mm}^{3}$, and platelet count was $78,000 /$ $\mathrm{mm}^{3}$ on Day 0 and $30,000 / \mathrm{mm}^{3}$ on Day 2. Peripheral blood smear (PBS) showed microcytic hypochromic anemia with tear drop cells and thrombocytopenia. Micro-hematuria was detected on urine routine examination. His liver and renal function tests were within normal limits. Tube Widal test titers were $<1: 40$ for TO, TH, and AH. Serum RK-39 was negative. Chinkungunya immunoglobulin M enzyme-linked immunosorbent assay was negative. Dengue immunoglobulin M enzyme-linked immunosorbent assay (NIV, Pune, India) was reactive. Rapid malaria antigen test (RMAT) (Ybio, Goa, India) on Day 0 was positive for $P$. vivax only. However, on PBS examination, rings of $P$. vivax and rings and gametocytes of $P$. falciparum were seen. Parasite density for P. falciparum was found to be $2 \%$ (Figure 1). Polymerase chain reaction (PCR) assay confirmed the presence of both the species (P. falciparum and $P$. vivax) in the patient. Abdominal ultrasound showed enlarged spleen (30 $\mathrm{cm}$ in size), mild hepatomegaly, and ascites. Bilateral pleural effusion was also noted.

A final diagnosis of mixed malarial infection (P. vivax and $P$. falciparum) with dengue fever coinfection with pancytopenia was made.

The patient was prescribed intravenous (IV) artesunate $2.4 \mathrm{mg} / \mathrm{kg}$ on Day 0 then at $12 \mathrm{~h}$ and $24 \mathrm{~h}$. He was also administered IV ceftriaxone $600 \mathrm{mg}$ b.d., IV fluids, IV paracetamol, and IV ondansetron and pantoprazole as supportive management on Day 0 of admission. The next day, the patient was switched to oral ACT (1 tablet of
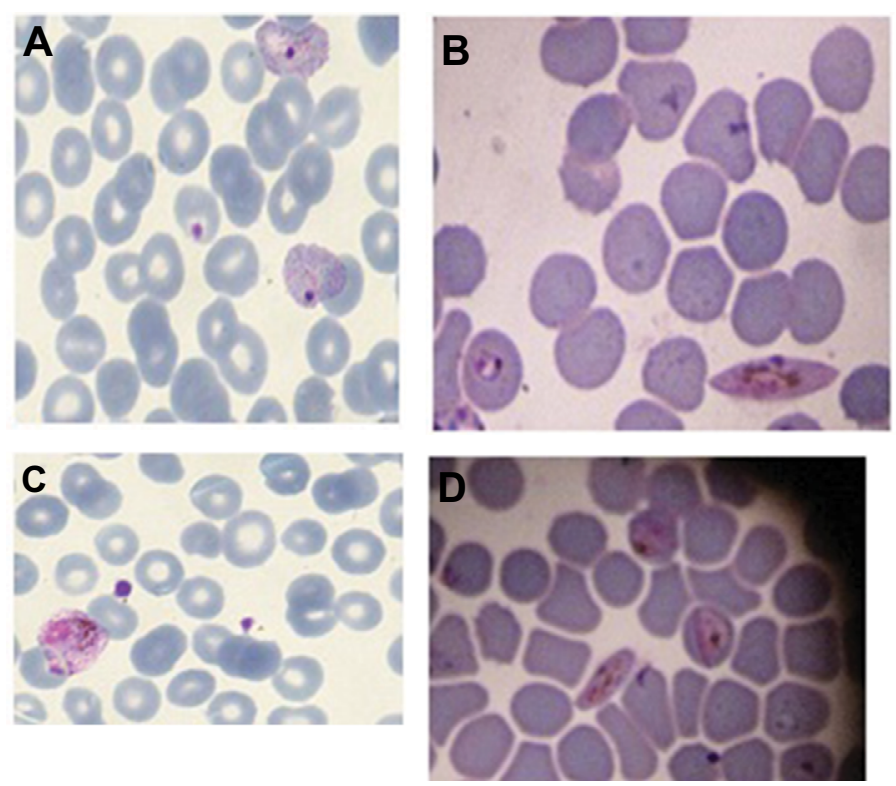

Figure I Asexual stages of malaria parasite in PBS (Day 0). (A) Ring forms of $P$. falciparum and $P$. vivax. (B) Ring form and gametocytes of $P$. falciparum. Day 3 - PBS. (C) Ruptured schizonts of $P$. vivax. (D) Rings, accole forms, and gametocytes of $P$. falciparum.

Abbreviation: PBS, peripheral blood smear. 
artesunate $200 \mathrm{mg}$ and 2 tablets of sulfadoxine/pyrimthamine $750 \mathrm{mg} / 37.5 \mathrm{mg}$ on Day 1 followed by artesunate $200 \mathrm{mg}$ for next 2 days).

By Day 3 of treatment, the patient's condition had improved, but fever persisted $\left(>37.5^{\circ} \mathrm{C}\right)$.

RMAT and PBS were reperformed on Day 3. On PBS, ruptured schizonts of $P$. vivax, rings and gametocytes of $P$. falciparum were seen, with a rise in parasitemia to $5 \%$. (Figure 1). RMAT was now positive for both $P$. vivax and $P$. falciparum.

Unfortunately, the patient left the hospital against medical advice and was lost to follow-up.

As the patient had rise in parasitemia with fever of $\left(>37.5^{\circ} \mathrm{C}\right)$ on Day 3 of treatment, he was diagnosed as a case of early treatment failure (ETF) according to the National Vector Borne Diseases Control Programme guidelines. ${ }^{3}$ The patient's blood spots were inoculated on Whatman filter paper no. 1 for further molecular analysis.

Genomic DNA extraction of the patient sample collected on Day 0 and Day 3 was done from filter paper blood spots using a QIAamp DNA Blood Mini Kit (QiagenInc., Hilden, Germany) according to the manufacturer's instructions. Diagnostic confirmation of Plasmodium species was done by $18 \mathrm{~S}$ rRNA PCR assay, which confirmed the results of microscopy and RMAT, showing mixed infections of $P$. falciparum and $P$. vivax in the patient (Figure 2). The samples were also genotyped for the drug resistance genes viz: chloroquine resistance ( $p f c r t$ ), multidrug resistance (pfmd-rl), sulfadoxine (pfdhps), pyrimethamine (pfdhfr), and artemisinin resistance (keltch 13). Single-nucleotide polymorphisms (SNPs) in pfcrt, pfmdr1, pfdhfr, pfdhps, and keltch 13 genes were assessed by PCR-RFLP and sequencing as described previously. ${ }^{4-6}$ Restriction site for ApoI enzyme for K76T mutation associated with chloroquine resistance in the $p f c r t$ gene and N86Y SNP for restriction enzyme AfIII in $p f m d r-1$ gene were seen in both the samples of the patient (Figure 2). Two mutations in pfdhfr gene at C59R and S108N were observed and there was one mutation in the pdhps gene at A437G. One SNP was seen on aligning with wild-type sequences of pfdhfr (GenBank accession no. 9221804) and pfdhps (GenBank accession no. 2655294) using Clustal W of the BioEdit 7.0 and MEGA (Molecular Evolutionary Genetics Analysis; http://www.megasoftware.net/) 4.0 programs (Table 1$).{ }^{7}$ Since this case was diagnosed as a case of ETF and artesunate resistance was suspected, sequence analysis to detect the presence of any SNP in kelch13 was also carried out. However, no SNPs were detected in this region (Table 1).

\section{Discussion}

The prevalence of mixed malarial infections, ie, concurrent infections with two or more malaria species have been reported as being in a range of $13.2 \%-45 \%$ by various studies from India. ${ }^{8-10}$ However, these studies were conducted in high-transmission areas in the country where the chances of acquiring mixed infections are more. One such study evaluating the clinical effect of mixed malarial infections suggested that mixed infection can present with severe manifestations in comparison with $P$. vivax infection alone; however, $P$. vivax coinfection has a protective role over $P$. falciparum
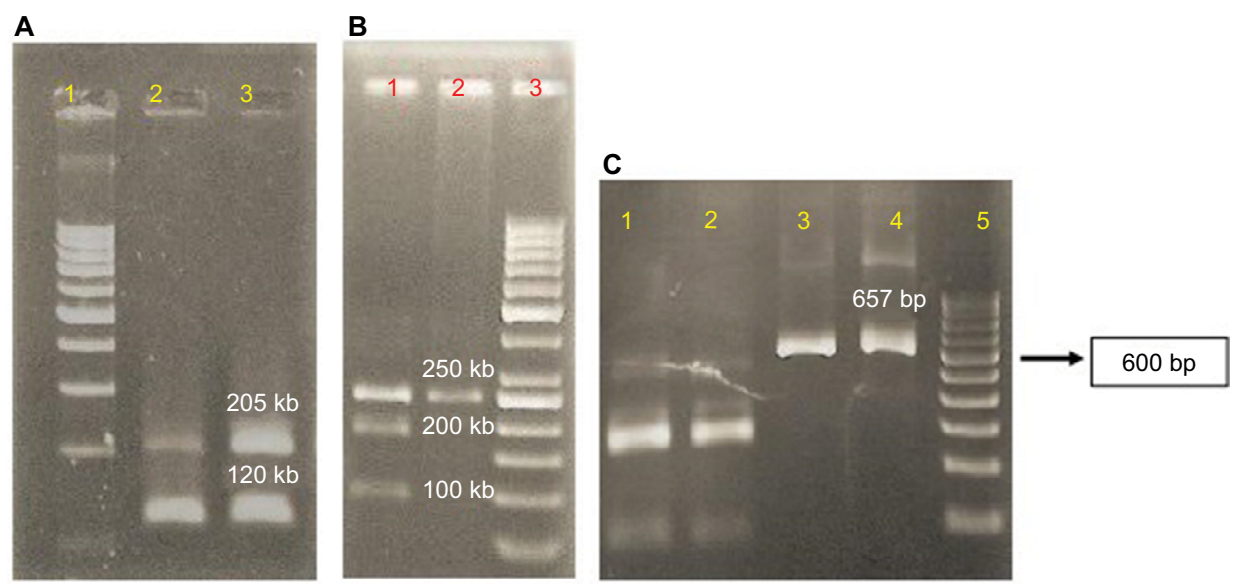

Figure 2 (A) I8S PCR results showing mixed infection inpatient samples. Lane I contains 100 bp ladder, lane 2 has Day 0 sample, and lane 3 has Day 3 sample. Both samples show mixed infections of $P$. falciparum at 205 bp and $P$. vivax at $I 20$ bp, respectively. (B) RE analysis of pfcrt gene. Lane I contains Day 0 sample and lane 2 contains Day 3 sample with $50 \mathrm{bp}$ ladder in lane 3. (C) Restriction digestion of pfmdr-I amplicon. Lanes I and 2 are patient samples confirming the SNP (N86Y) in the amplicons (lanes I and 2 are Day 0 and Day 3 sample, respectively), lanes 3 and 4 are undigested amplicons, and lane 5 contains 100 bp ladder. Abbreviations: PCR, polymerase chain reaction; RE, restriction enzyme; SNP, single-nucleotide polymorphism. 


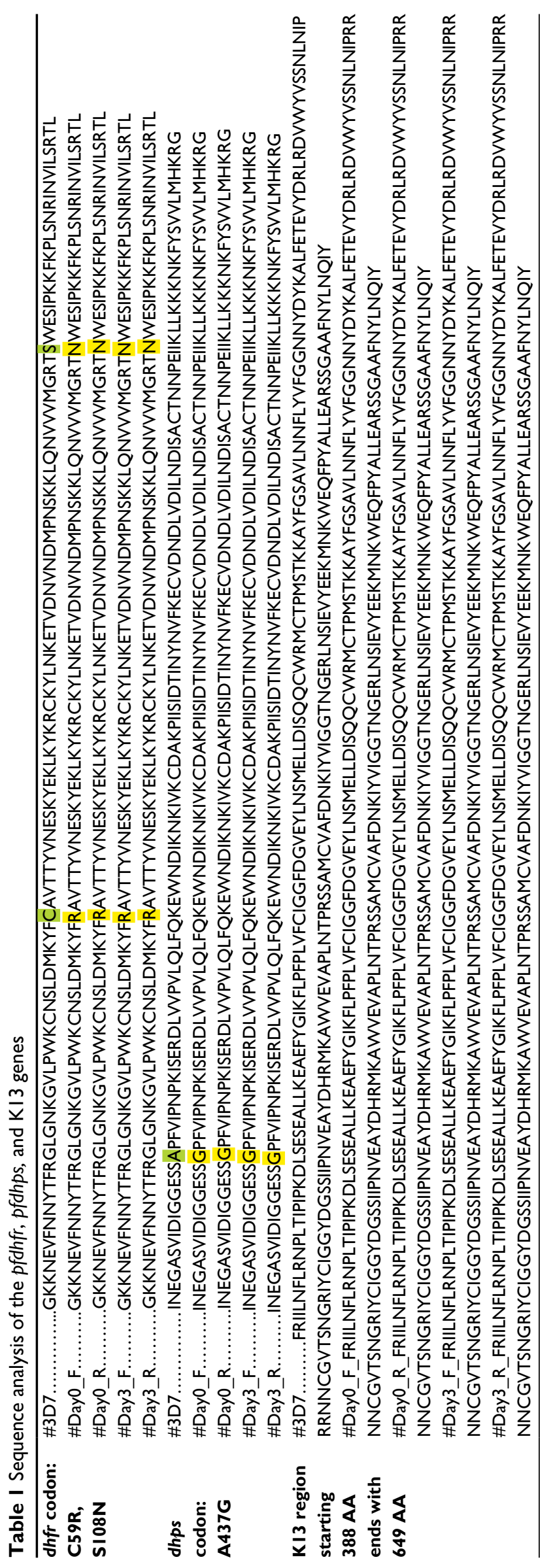

monoinfection. ${ }^{10}$ The percentage of mixed species infection and its effect on patient's clinical course in low-transmission region like Northern India, especially Delhi, are not known.

Dengue and malaria are two most common vector-borne diseases in India. Both diseases have similar geographical (tropical and subtropical regions) and seasonal distribution (June-December). ${ }^{11,12}$ Cases of coinfection of dengue and malaria have recently been reported by various studies from India, with rates of coinfection ranging from $<1 \%$ to $10.25 \% \cdot{ }^{13-15}$ Delhi is known to be highly endemic for dengue fever; however, it is a low-transmission area for malarial parasites. Rapid urbanization, high population density, unhygienic conditions in urban slums, and open drains have resulted in breeding of mosquitoes, both Aedes and Anopheles spp. Our patient lived in a slum with a drain near his house and had been exposed to multiple mosquito vectors. The studies on impact of dengue-malaria coinfection on clinical and laboratory parameters have shown that individuals with dual infections display lower levels of platelets and increased risk of hepatomegaly, jaundice, and severe anemia but that other features of severe disease are comparable or lower. ${ }^{11,15,16}$ In the present case, concurrent infection with malaria and dengue due to mixed species resulted in severe illness with anemia and splenomegaly. It has been observed in studies that the clinical features of concurrent infection were more like dengue monoinfection than malaria monoinfection. ${ }^{8}$ Therefore, it is necessary to screen for malaria in patients with dengue for diagnosis of such cases.

Though the impact of coinfections on treatment failure have been not reported as yet, our patient had a rise in parasitemia with fever of $>37.5^{\circ} \mathrm{C}$ on Day 3 of treatment, and thus showed signs of ETF. According to the National Vector Borne Diseases Control Programme guidelines, ETF in malaria is defined as development of danger signs or severe malaria on Day 1,2, or 3 in the presence of parasitemia; parasitemia on Day 2 higher than on Day 0, irrespective of axillary temperature; parasitemia on Day 3 with axillary temperature $>37.5^{\circ} \mathrm{C}$; and parasitemia on Day 3, $>25 \%$ of count on Day $0 .{ }^{3}$ This could be attributed to either inappropriate treatment or drug resistance. Since incorrect dosing, noncompliance with duration of dosing regimen, poor drug quality, drug interactions, poor or erratic absorption, and misdiagnosis were ruled out, we suspected drug resistance to be the cause of ETF.

The samples collected on Day 0 and Day 3 both revealed mutations in the drug resistance genes. The K76T mutation in pfcrt gene is the primary mediator of chloroquine resistance. ${ }^{17}$ P. falciparum strains with higher number of alleles of $p f m d r-$ lgene and SNPs like N86Y are associated with chloroquine resistance. ${ }^{18}$ These SNPs in $p f c r t$ and $p f m d r-1$ genes also result 
in reduced susceptibilities to partner drugs in ACT like lumefantrine and meflaquine. ${ }^{18}$ The $p f m d r-1$ gene has been associated with altered susceptibilities of trophozoite-stage parasites to artemisinin derivatives. ${ }^{19}$ The parasitic strain isolated from the patient also showed two mutations in pfdhfr gene (C59R, $\mathrm{S} 108 \mathrm{~N})$ and one mutation in $p f d h p s$ gene (A437G). The presence of C59R along with S108N mutations in pfdhfr confer a significant resistance to pyrimethamine, and $\mathrm{A} 437 \mathrm{G}$ mutation is associated with resistance to sulfadoxine. ${ }^{18} \mathrm{~A}$ rise in such triple mutations have been reported from Iran 7 years after adoption of sulfadoxine+ pyrimethamine as first-line treatment in $\mathrm{ACT} .^{20}$ $\mathrm{K} 13$ propeller gene mutations have been linked to both in vitro artemisinin resistance and in vivo slow parasite clearance rates. ${ }^{17}$ Though no sequence mutation was seen in keltch13, the presence of several other SNPs and concurrent dengue infection may have been responsible for ETF in our patient.

\section{Conclusion}

The present case highlights the complex ways in which mosquito-borne diseases can present in areas of common endemicities. Though mutations in K13 gene were not detected in this case, further studies are required to understand the cumulative effect of mutations detected in multiple genes and concurrent dengue infection in this case. There is a need for constant monitoring of efficacy of ACT as the misuse of sulfadoxine+pyrimthamine tablets available as over-the-counter drugs may lead to widespread resistance and exposure of artemisinin derivatives as monotherapy. In our resource-limited setting, where molecular analysis of all samples is not possible, we recommend that determination of parasitemia by peripheral blood examination must accompany RMAT results and that a parasite count must be done when the patient is discharged to look for cases of treatment failure.

\section{Limitations}

The patient left against medical advice on Day 3 of treatment, and so follow-up for late therapeutic response or any relapse could not be monitored.

\section{Author contributions}

RS and MM conceived the study; DK, MM, and AA carried out the clinical assessment; MM and RS carried out the malaria and dengue testing; VS and AK carried out the molecular analysis; RS, MM, and VS drafted the manuscript; RS, MM, and RG critically revised the final manuscript. All authors contributed toward data analysis, drafting and critically revising the paper and agree to be accountable for all aspects of the work. RS and MM are guarantors of the paper.

\section{Disclosure}

The authors report no conflicts of interest in this work.

\section{References}

1. NVBDCP. National Framework for Malaria Elimination in India. Delhi: Directorate of National Vector Borne Disease Control Program; 2015.

2. NVBDCP. Dengue Cases and Deaths in the Country Since 2010. Delhi: Directorate of National Vector Borne Disease Control Program; 2016

3. NVBDCP. Guidelines for Diagnosis and Treatment of Malaria in India. Delhi: Directorate of National Vector Borne Disease Control Program; 2011.

4. Gatton ML, Martin LB, Cheng Q. Evolution of resistance to sulfadoxine-pyrimethamine in Plasmodium falciparum. Antimicrob Agents Chemother. 2004;48(6):2116-2123.

5. Khatoon L, Ballraine FN, Malik SA, Yan G. Sequence analysis of genes associated with resistance to chloroquine and sulphadoxine pyrimethamine in P. falciparum and P. vivax isolates from the Bannu district of Pakistan. Braz J Infect Dis. 2013;17(5):596-600.

6. Ekland EH, Fidock DA. Advances in understanding the genetic basis of antimalarial drug resistance. Curr Opin Microbiol. 2007;10(4):363-370.

7. Chenna R, Sugawara H, Koike T, et al. Multiple sequence alignment with the clustal series of programs. Nucleic Acids Res. 2003;31:3497-3500.

8. Mohapatra MK, Dash LK, Barih PK, Karua PC. Profile of mixed species (Plasmodium vivax and falciparum) malaria in adults. $J$ Assoc Physicians India. 2012;60:20-24.

9. Gupta B, Gupta P, Sharma AA, Singh V, Dash AP, Das A. High proportion of mixed-species Plasmodium infections in India revealed by PCR diagnostic assay. Trop Med Int Health. 2010;15:819-824.

10. Krishna S, Bharti PK, Chandel HS, et al. Detection of mixed infections with Plasmodium spp. by PCR, India, 2014. Emerg Infect Dis. 2015;21(10):1853-1857.

11. Mendonça VR, Andrade BB, Souza LC, et al. Unravelling the patterns of host immune responses in Plasmodium vivax malaria and dengue coinfection. Malar J. 2015;14:315.

12. Rao MRK, Padhy RN, Das MK. Prevalence of dengue viral and malaria parasitic co-infections in an epidemic district, Angul of Odisha, India: an eco-epidemiological and cross-sectional study for the prospective aspects of public health. $J$ Infect Public Health. 2016;9:421-428.

13. Singh R, Singh SP, Ahmad N. A study of etiological pattern in an epidemic of acute febrile illness during monsoon in a tertiary health care institute of Uttarakhand, India. J Clin Diagn Res. 2014;8(6):MC01-MC03.

14. Barua A, Gill N. A comparative study of concurrent dengue and malaria infection with their monoinfection in a teaching hospital in Mumbai. $J$ Assoc Physicians India. 2016;64(8):49-52.

15. Mohapatra MK, Patra P, Agrawala R. Manifestation and outcome of concurrent malaria and dengue infection. J Vector Borne Dis. 2012;49:262-265.

16. Arumugam JMR, Mary A, Satagopan U. A review on coinfection with dengue and malaria. Int J Trop Med Public Health. 2015;5(3):1-5.

17. Cui L, Mharakurwa S, Ndiaye D, Rathod PK, Rosenthal PJ. Antimalarial drug resistance: literature review and activities and findings of the ICEMR network. Am J Trop Med Hyg. 2015;93: 57-68.

18. Ibraheem ZO, Abd Majid R, Noor SM, Sedik HM, Basir R. Role of different Pfcrt and Pfmdr-1 mutations in conferring resistance to antimalaria drugs in Plasmodium falciparum. Malar Res Treat. 2014;2014: Article ID 950424.

19. Veiga MI, Dhingra SK, Henrich PP, et al. Globally prevalent PfMDR1 mutations modulate Plasmodium falciparum susceptibility to artemisinin-based combination therapies. Nat Commun. 2016;7: Article no 11553.

20. Afsharpad M, Zakeri S, Pirahmadi S, Djadid ND. Molecular monitoring of Plasmodium falciparum resistance to antimalarial drugs after adoption of sulfadoxine-pyrimethamine plus artesunate as the first line treatment in Iran. Acta Trop. 2012;121:13-18. 
The International Medical Case Reports Journal is an international, peer-reviewed open-access journal publishing original case reports from all medical specialties. Previously unpublished medical posters are also accepted relating to any area of clinical or preclinical science. Submissions should not normally exceed 2,000 words or
4 published pages including figures, diagrams and references. The manuscript management system is completely online and includes a very quick and fair peer-review system, which is all easy to use. Visit http://www.dovepress.com/testimonials.php to read real quotes from published authors.

Submit your manuscript here: https://www.dovepress.com/international-medical-case-reports-journal-journal 